\title{
A New Approach for the Stability Analysis in Hydromagnetic Couette Flow
}

\author{
Adjimon Vincent Monwanou1, Amoussou Laurent Hinvi², Hodévèwan Clément Miwadinou³, \\ Jean Bio Chabi Orou ${ }^{1}$ \\ ${ }^{1}$ Institut de Mathématiques et de Sciences Physiques, Porto-Novo, Bénin \\ ${ }^{2}$ Institut Universitaire de Technologie, Lokossa, Bénin \\ ${ }^{3}$ Ecole Normale Supérieure, Natitingou, Bénin \\ Email: vincent.monwanou@imsp-uac.org, laurent.hinvi@imsp-uac.org, clement.miwadinou@imsp-uac.org, jchabi@yahoo.fr
}

How to cite this paper: Monwanou, A.V., Hinvi, A.L., Miwadinou, H.C. and Chabi Orou, J.B. (2017) A New Approach for the Stability Analysis in Hydromagnetic Couette Flow. Journal of Applied Mathematics and Physics, 5, 1503-1514.

https://doi.org/10.4236/jamp.2017.57123

Received: June 26, 2017

Accepted: July 25, 2017

Published: July 28, 2017

Copyright (c) 2017 by authors and Scientific Research Publishing Inc. This work is licensed under the Creative Commons Attribution International License (CC BY 4.0)

http://creativecommons.org/licenses/by/4.0/

\begin{abstract}
This paper analyses the effects of small injection/suction Reynolds number, Hartmann parameter, permeability parameter and wave number on a viscous incompressible electrically conducting fluid flow in a parallel porous plates forming a channel. The plates of the channel are parallel with the same constant temperature and subjected to a small injection/suction. The upper plate is allowed to move in flow direction and the lower plate is kept at rest. A uniform magnetic field is applied perpendicularly to the plates. The main objective of the paper is to study the effect of the above parameters on temporal linear stability analysis of the flow with a new approach based on modified Orr-Sommerfeld equation. It is obtained that the permeability parameter, the Hartmann parameter and the wave number contribute to the linear temporal stability while the small injection/suction Reynolds number has a negligible effect on the stability.
\end{abstract}

\section{Keywords}

Hydromagnetic Flat Couette Flow, Injection/Suction Parameter, Modified Orr-Sommerfeld Equation, Temporal Linear Stability

\section{Introduction}

The study of Couette flow in a rectangular channel of an electrically conducting viscous fluid under the action of a transversely applied magnetic field has immediate applications in many devices such as magnetohydrodynamic (MHD) power generators, MHD pumps, accelerators, aerodynamics heating, electrostatic precipitation, polymer technology, petroleum industry, purification of crude oil and fluid droplets spray. Channel flows of a Newtonian fluid with or without heat transfer were studied with or without Hall currents by many authors [1]-[8]. 
The effects of the injection/suction through the injection/suction parameter number, Hartmann parameter, permeabilty or Darcy parameter on the stability of the fluids flows were also studied with different approach [2] [5] [6] [7] [8].

Indeed, the heat source and the Soret effect on hydromagnetic oscillatory flow through a porous medium bounded by two vertical parallel porous plates have been analyzed by Chand [5], where one plate of the channel is kept stationary and the other is moving with uniform velocity. The plates of the channel are subjected to constant injection and suction velocities respectively. They remarked that the Lorentz force parameter i.e. the Hartmann number contributes to reduce the velocity and the skin friction profiles. The effect of the permeability parameter i.e. Darcy number is just opposite to that of Lorentz force parameter. Nayak et al. [8] studied an oscillatory effect on magnetohydrodynamic flow and heat transfer in rotating horizontal porous channel. They found that magnetic field fixed relative to the moving plate contributes more to the resultant velocity than the magnetic field fixed relative to the fluid in case of all the parameters. Another striking result is that frequency of oscillation has a distinct effect when the magnetic field is fixed relative to the fluid. The effect of all the pertinent parameters on phase angle is just opposite to that of resultant velocity owing to the relative positions of the magnetic fields. In [6], the authors have considered the unsteady hydromagnetic incompressible viscous fluid flow through a porous medium in a horizontal channel under prescribed discharge, under the influence of inclined magnetic field. Das [2] analyzed the effects of constant suction and sinusoidal injection on three dimensional Couette flow of a viscous incompressible electrically conducting fluid through a porous medium between two infinite horizontal parallel porous flat plates in presence of a transverse magnetic field. The stationary plate and the plate in uniform motion are, respectively, subjected to a transverse sinusoidal injection and uniform suction of the fluid. It is observed that a growing magnetic parameter retards the main velocity and accelerates the cross flow velocity of the flow field and a growing permeability parameter or suction/injection parameter reverses the effect. Both Prandtl number and the suction/injection parameter have retarding effect on the temperature field. Further, a growing suction/injection parameter diminishes both the components of skin friction at the wall while the permeability parameter enhances the $x$-component and reduces the $z$-component of the skin friction at the wall. In [7], the authors studied the effects of variable viscosity and heat source on unsteady laminar flow of dusty conducting fluid between parallel porous plates through porous medium with temperature dependent viscosity. It is assumed that the parallel plates are porous and subjected to a uniform suction from above and injection from below. They found that the effect of the suction velocity on both the velocity and temperature of the fluid and particles is more pronounced for higher values of the porosity parameter.

In the present paper, we studied the effects of above parameters on the linear temporal stability of the fluid, in a Couette horizontal porous channel flow with the presence of a uniform transverse magnetic field fixed relative to the fluid. We 
used a new approach based on a derived equation named modified OrrSommerfeld equation. The corresponding eigenvalue problem is resolved in order to study the linear stability of the flow. The plates of the channel are considered porous and flow within the channel is due to the uniform motion of the upper plate. Such linear temporal stability analysis through the so-called modified Orr-Sommerfeld equation has been made earlier [4] in a Poiseuille flow without injection/suction. The authors showed that the magnetic field has a stabilizing effect on the electrically conducting fluid flows.

The paper is organized as follows: Sect. 2 addresses the so-called modified Orr-Sommerfeld equation governing the stability analysis in the hydromagnetic Couette horizontal porous plates flow. Section 3 deals with analysis of the effects of small injection/suction Reynolds number $R_{e \omega}$, Hartmann parameter $M$, wave number $k$ and permeability parameter $K_{p}$ on the flow. The conclusion is presented in the last section.

\section{Modified Orr-Sommerfeld Equation}

We considere a Couette viscous incompressilbe, electrically conducting fluid flow between two porous parallel plates of infinite lengh, distant $h$ apart in the presence of uniform transverse constant magnetic field $B_{0}$ applied parallel to $y^{*}$ axis which is normal to the planes of the plates. We considered the simple case where, $B_{0}$ is fixed relative to the fluid. We work at constant temperature, the heat transfer aspect of the flow is not studied. We applied a small constant injection $V_{\omega}$, at the lower plate and a same small constant suction $V_{\omega}$, at the upper plate. The upper plate is allowed to move with non-zero uniform velocity $U=U_{0}$ in flow direction and the lower plate is kept at rest. We choose the origine on the plane $\left(x^{*}, 0, z^{*}\right)$ such as $-h \leq y^{*} \leq h$ and $x^{*}$ parallel to the direction of the motion of the upper plate. We assumed the magnetic Reynolds number very small for metallic liquids and neglected the induced magnetic field in comparison with the applied one [3] [7] [8]. Initially, $t^{*}=0$, both the fluid and plates are assumed to be at rest. When $t^{*}>0$, the upper plate starts moving with a constant velocity $U$ in coordinate system with the fluid. The equations of continuity, motion for the viscous incompressible electrically conducting fluid in vector form are:

$$
\begin{gathered}
\nabla \cdot V=0, \\
\frac{\partial V}{\partial t^{*}}+(V \cdot \nabla) V=-\frac{1}{\rho} \nabla p+\nu \nabla^{2} V+\frac{1}{\rho} J \wedge B-\frac{\mu V}{\rho k^{*}}, \\
\nabla \wedge B=\mu_{e}\left(J+\varepsilon_{e} E\right), \\
\nabla \wedge E=-\frac{\partial B}{\partial t^{*}}, \\
\nabla \cdot B=0, \\
\nabla \cdot E=0, \\
\nabla \cdot J=0,
\end{gathered}
$$




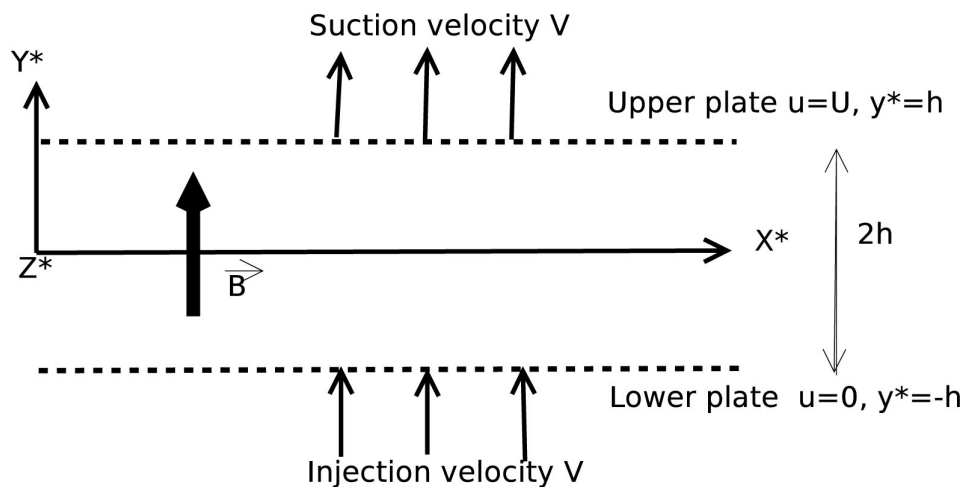

Figure 1. Physical model and coordinate system.

where (1)-(7) are continuity, Newton's second law, Ampere's law, Faraday's law, Maxwell's law and Gauss law equations respectively, with

$$
J=\sigma(E+V \wedge B),
$$

and $V\left(u^{*}, v^{*}, w^{*}\right), B, E, J, \sigma, \mu_{e}, \varepsilon_{e}$ are the velocity, the magnetic field, the electric field, the current density vector, the fluid electrical conductivity, the magnetic permeability and absolute permittivity of the fluid respectively and $t^{*}$ denotes the time. The physical model of the problem is illustrated in Figure 1, where $V\left(u^{*}, v^{*}, w^{*}\right)$ is the velocity vector in the $x^{*}, y^{*}, z^{*}$ directions respectively.

$$
\begin{gathered}
B=\left(0, B_{0}, 0\right), \\
E=\left(E_{x}, E_{y}, E_{z}\right), \\
J=\left(J_{x}, 0, J_{z}\right) ;
\end{gathered}
$$

where $B_{0}$ is a constant. We assumed that no applied polarization voltage exists (i.e., $E=0$ ). Then Equation (8) and Equation (11) give

$$
J=\sigma B_{0}(-w, 0, u)
$$

and Equation (7) yields

$$
\sigma B_{0}\left(\frac{\partial u}{\partial z}-\frac{\partial w}{\partial x}\right)=0
$$

We introduce the following non-dimensional quantities $\tilde{x}=\frac{x^{*}}{h}, \quad \tilde{y}=\frac{y^{*}}{h}$, $\tilde{z}=\frac{z^{*}}{h}, \quad \tilde{t}=\frac{U t^{*}}{h}, \quad \tilde{u}=\frac{u^{*}}{U}, \quad \tilde{v}=\frac{v^{*}}{V_{\omega}}, \quad \tilde{w}=\frac{w^{*}}{U}, \quad \tilde{p}=\frac{p^{*}}{\rho U^{2}}, \quad R_{e}=\frac{U h}{v}$ (hydrodynamic Reynolds number), $R_{e \omega}=\frac{V_{\omega} h}{v}$ (injection/suction Reynolds number), $\quad M=B_{0} h \sqrt{\frac{\sigma}{\mu}} \quad$ (Hartmann parameter), $\quad K_{p}=\frac{k^{*}}{h^{2}} \quad$ (permeability parameter).

So, Equation (1) and Equation (2) become

$$
\frac{\partial \tilde{u}}{\partial \tilde{x}}+\frac{R_{e \omega}}{R_{e}} \frac{\partial \tilde{v}}{\partial \tilde{y}}+\frac{\partial \tilde{w}}{\partial \tilde{z}}=0
$$




$$
\begin{gathered}
\frac{\partial \tilde{u}}{\partial \tilde{t}}+\tilde{u} \frac{\partial \tilde{u}}{\partial \tilde{x}}+\frac{R_{e \omega}}{R_{e}} \tilde{v} \frac{\partial \tilde{u}}{\partial \tilde{y}}+\tilde{w} \frac{\partial \tilde{u}}{\partial \tilde{z}}=-\frac{\partial \tilde{p}}{\partial \tilde{x}}+\frac{\nabla^{2} \tilde{u}}{R_{e}}-\frac{M^{2}}{R_{e}} \tilde{u}-\frac{\tilde{u}}{K_{p} R_{e}}, \\
\frac{\partial \tilde{v}}{\partial \tilde{t}}+\tilde{u} \frac{\partial \tilde{v}}{\partial \tilde{x}}+\frac{R_{e \omega}}{R_{e}} \tilde{v} \frac{\partial \tilde{v}}{\partial \tilde{y}}+\tilde{w} \frac{\partial \tilde{v}}{\partial \tilde{z}}=-\frac{R_{e}}{R_{e \omega}} \frac{\partial \tilde{p}}{\partial \tilde{y}}+\frac{\nabla^{2} \tilde{v}}{R_{e}}-\frac{\tilde{v}}{K_{p} R_{e}}, \\
\frac{\partial \tilde{w}}{\partial \tilde{t}}+\tilde{u} \frac{\partial \tilde{w}}{\partial \tilde{x}}+\frac{R_{e \omega}}{R_{e}} \tilde{v} \frac{\partial \tilde{w}}{\partial \tilde{y}}+\tilde{w} \frac{\partial \tilde{w}}{\partial \tilde{z}}=-\frac{\partial \tilde{p}}{\partial \tilde{z}}+\frac{\nabla^{2} \tilde{w}}{R_{e}}-\frac{M^{2}}{R_{e}} \tilde{w}-\frac{\tilde{w}}{K_{p} R_{e}} .
\end{gathered}
$$

For the stability analysis, the flow is decomposed into the mean flow and the disturbance according to

$$
\begin{gathered}
\tilde{u}_{i}(r, t)=U_{i}(r)+u_{i}(r, t), \\
\tilde{p}(r, t)=P(r)+p(r, t) .
\end{gathered}
$$

We take the dimensional basic flow for small suction and injection [2] [4] [5],

$$
\begin{gathered}
U^{*}(y)=\frac{U}{2}\left(\frac{y^{*}}{h}+1\right), \\
V^{*}=V_{\omega}, \\
W^{*}=0 .
\end{gathered}
$$

By scaling these velocities as above, we obtain with $h= \pm 1 \quad\left(-1 \leq y^{*} \leq 1\right)$ the no-dimensional base flow

$$
\begin{aligned}
U(y) & =\frac{y+1}{2}, \\
V & =1, \\
W & =0 .
\end{aligned}
$$

To obtain the stability equations for the spatial evolution of three-dimensional, we take the dependent on time disturbances

$$
(u(x, y, z, t) ; v(x, y, z, t) ; w(x, y, z, t) ; p(x, y, z, t)) ;
$$

which are scaled in the same way as above.

Inserting Equations (18) (26) into Equations (15)-(17), we get

$$
\begin{gathered}
\frac{\partial u}{\partial t}+U \frac{\partial u}{\partial x}+\frac{R_{e \omega}}{R_{e}} \frac{\partial u}{\partial y}+\frac{R_{e \omega}}{R_{e}} v \frac{\partial U}{\partial y}=-\frac{\partial p}{\partial x}+\frac{\nabla^{2} u}{R_{e}}-\frac{M^{2}}{R_{e}} u-\frac{u}{K_{p} R_{e}}, \\
\frac{\partial v}{\partial t}+U \frac{\partial v}{\partial x}+\frac{R_{e \omega}}{R_{e}} \frac{\partial v}{\partial y}=-\frac{R_{e}}{R_{e \omega}} \frac{\partial p}{\partial y}+\frac{\nabla^{2} v}{R_{e}}-\frac{v}{K_{p} R_{e}}, \\
\frac{\partial w}{\partial t}+U \frac{\partial w}{\partial x}+\frac{R_{e \omega}}{R_{e}} \frac{\partial w}{\partial y}=-\frac{\partial p}{\partial z}+\frac{\nabla^{2} w}{R_{e}}-\frac{M^{2}}{R_{e}} w-\frac{w}{K_{p} R_{e}} .
\end{gathered}
$$

The pressure terms can be eliminated from Navier-Stokes equations. For such a mean profile (base flow), the divergence of Navier-Stokes equations and continuity, give

$$
\nabla^{2} p=-2 \frac{R_{e \omega}}{R_{e}} \frac{\mathrm{d} U}{\mathrm{~d} y} \frac{\partial v}{\partial x}+M^{2} \frac{R_{e \omega}}{R_{e}^{2}} \frac{\partial v}{\partial y} .
$$

Taking the laplacian of Equation (28), we get after linearization with Equation 
(30)

$$
\left[\frac{\partial}{\partial t}+U \frac{\partial}{\partial x}+\frac{R_{e \omega}}{R_{e}} \frac{\partial}{\partial y}+\frac{1}{K_{p} R_{e}}-\frac{\nabla^{2}}{R_{e}}\right] \nabla^{2} v-\frac{\mathrm{d}^{2} U}{\mathrm{~d} y^{2}} \frac{\partial v}{\partial x}+\frac{M^{2}}{R_{e}} \frac{\partial^{2} v}{\partial y^{2}}=0 .
$$

The disturbances are taken to be periodic in the streamwise, spanwise directions and time, which allow us to assume solutions of the form

$$
f(x, y, z, t)=\hat{f}(y) \mathrm{e}^{i(\alpha x+\beta z-\omega t)} ;
$$

where $f$ represents either one of the disturbances $u, v, w$ or $p$ and $\hat{f}$ the amplitude function; $\alpha=k_{x}=k \cos \theta$ and $\beta=k_{z}=k \sin \theta$ are the wave numbers respectively on $x$ and $z$ axis directions; $\omega=\alpha c$ is the frequency of the wave; $i^{2}=-1, \theta=\left(\boldsymbol{k}_{x}, \boldsymbol{k}\right), c=c_{r}+i c_{i}$ is the wave velocity which is taken to be complex, $\alpha$ and $\beta$ are real because of temporal stability analysis consideration. Then Equation (31) becomes

$$
i \alpha\left(U-c-i \frac{R_{e \omega} D}{\alpha R_{e}}-\frac{i}{\alpha K_{p} R_{e}}+i \frac{D^{2}-k^{2}}{\alpha R_{e}}\right)\left(D^{2}-k^{2}\right) \hat{v}=-\left(\frac{M^{2} D^{2}}{R_{e}}-i \alpha U^{\prime \prime}\right) \hat{v} ;
$$

where $D=\frac{\mathrm{d}}{\mathrm{d} y}$; with boundary conditions for all $(x, \pm 1, z, t>0)$

$$
\left\{\begin{array}{l}
\hat{v}( \pm 1)=1 \\
\hat{v}^{\prime}( \pm 1)=0
\end{array}\right.
$$

Taking

$$
v_{p}(x, y, z, t)=\hat{v}(y) \mathrm{e}^{i(\alpha x+\beta z-\omega t)}-1,
$$

Equation (33) and the boundary conditions Equation (34), take the forms

$$
\left\{\begin{array}{l}
{\left[\left(U-i \frac{R_{e \omega} D}{\alpha R_{e}}-\frac{i}{\alpha K_{p} R_{e}}+i \frac{D^{2}-k^{2}}{\alpha R_{e}}\right)\left(D^{2}-k^{2}\right)-\left(U^{\prime \prime}+\frac{i M^{2} D^{2}}{\alpha R_{e}}\right)\right] \hat{v}_{p}=c\left(D^{2}-k^{2}\right) \hat{v}_{p},} \\
\hat{v}_{p}( \pm 1)=\hat{v}_{p}^{\prime}( \pm 1)=0 .
\end{array}\right.
$$

The first equation of system Equation (36) is a flow equation modified by the small injection/suction Reynolds number $R_{e \omega}$, the Hartmann parameter $\left(M=B_{0} h \sqrt{\frac{\sigma}{\mu}}\right)$, and permeability parameter $\left(K_{p}=\frac{k^{*}}{h^{2}}\right)$ which is the so-called modified Orr-Sommerfeld equation, rewritten as an eigenvalue problem, where $c$ is the eigenvalue and $\hat{v}_{p}$ the eigenfunction.

\section{Linear Stability Analysis}

We consider a three-dimensional disturbances. We use a temporal stability analysis as mentioned above. With $c$ complex as defined above, when $c_{i}<0$, a stability mode takes place, $c_{i}=0$ corresponds to neutral stability and elsewhere corresponds to instability. We employ Matlab 7.8.0.(R2009a) version in all our numerical computations to find the eigenvalues. The Couette horizontal porous plates flow with the basic velocity profile 


$$
\mathbf{U}=\left(\frac{y+1}{2}, 1,0\right)
$$

for $R_{e \omega}$ small (i.e. small suction) is considered. The eigenvalue problem Equation (36) is solved numerically with the suitable boundary conditions. The solutions are found in a layer bounded at $y= \pm 1$ with $\mathbf{U}( \pm 1)=(0,1,0)$. The results of calculations are presented in the figures below.

The black, red, green and blue colors are respectively, curves I, II, III, IV and the yellow color figure corresponds to the neutral mode $c_{i}=0$. Frame $a, b, c, d$ correspond respectively to $k=1 \quad k=1.02, k=2$ and $k=3$.

Figure 2 presents the effect of injection/suction Reynolds number $R_{e \omega}$ on linear temporal stability of viscous incompressible electrically non conducting fluid ( $M=0$ ) flow for different values of wave number. It is observed that for $k=1$ and $k=1.02$ (frames a and b), the stability is not affectd by $R_{e \omega}$ and the flow is unstable but for $k=2$ and $k=3$ (frames $\mathrm{c}$ and d), $R_{e \omega}$ affects the stability, and the flow stays stable, but increasing of $R_{e \omega}$ doesn't contribute to the stability.

Figure 3 exhibits the effect of permeabilty parameter $K_{p}$ on linear temporal stability of viscous incompressible electrically non conducting fluid flow for different values of wave number. It is observed that $K_{p}$ affects the stability. For $k=1$ and $k=1.02$, the frames $a$ and $b$ show that for $K_{p}=0.045$ the flow is unstable (curves I) and stable for $K_{p}=1.000$ (curves IV) but for $K_{p}=0.048$ and $K_{p}=0.130$ (curves II, III), we have the transition of the flow (See Table 1 for the criticals hydrodynamic Reynolds number values). For $k=2$ and $k=3$ (frames $c$ and $d$ ) the flow is completely stable. On careful observation, we remark that for $R_{e}<12500$, increasing of $K_{p}$ contributes to the stability in frame $d$ case, and the opposite is noticed in the frame $c$ case, but when $R_{e}>12500$, increasing of $K_{p}$ contributes in the both cases. Thus, it
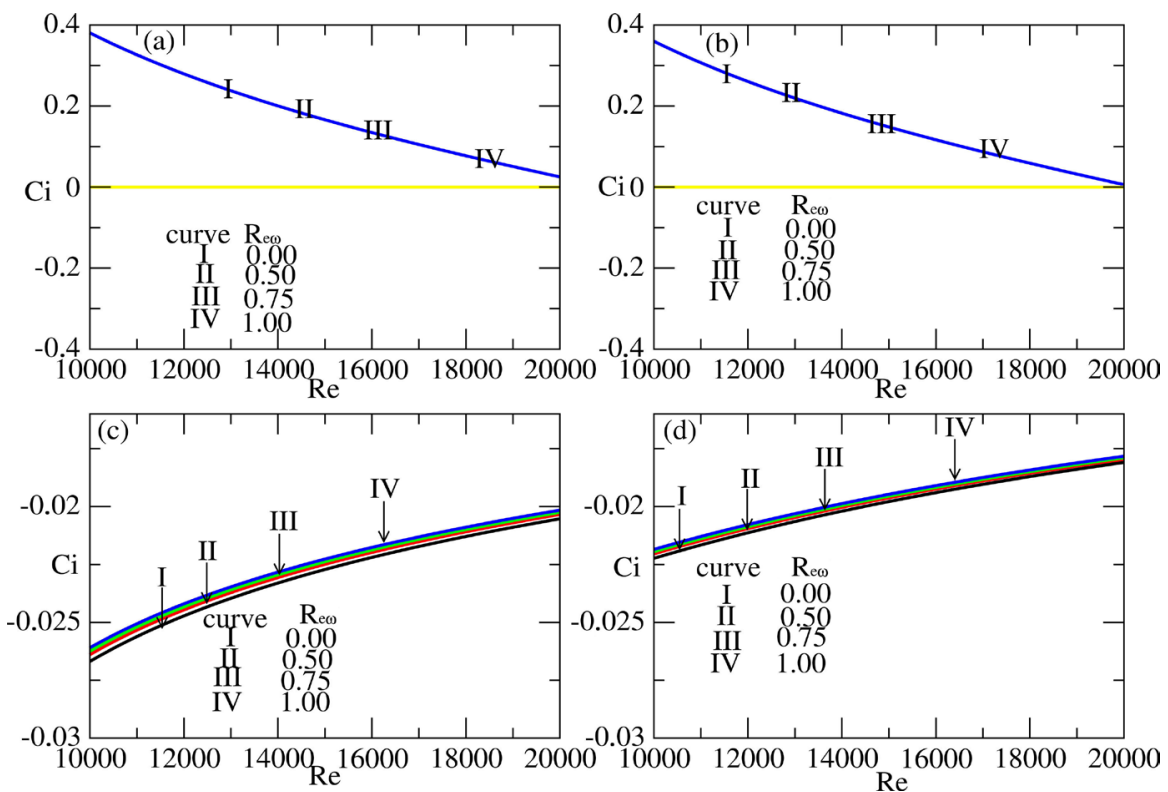

Figure 2. Ci vs. $\operatorname{Re}$ for $M=0, K_{p}=0, \theta=0$ and $R_{e \omega}, k$ variable. 

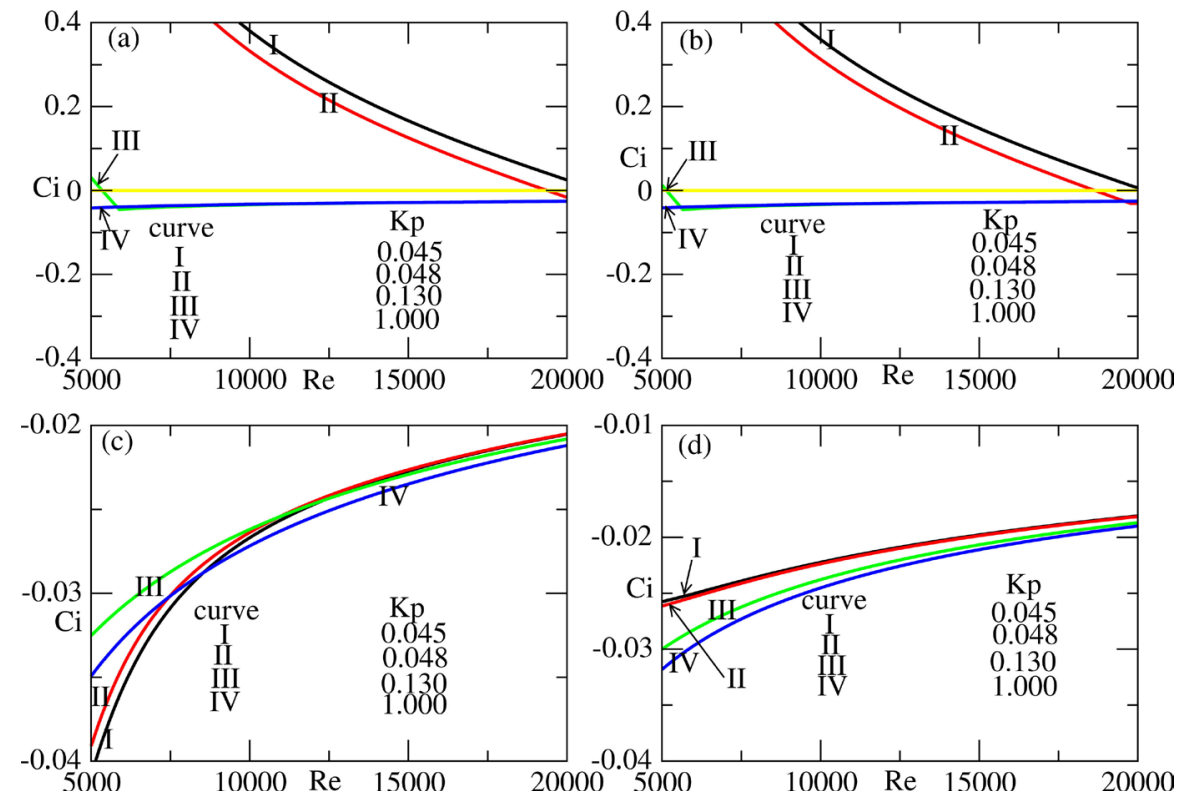

Figure 3. Ci vs. Re for $M=0, R_{e \omega}=0, \theta=0$ and $K_{p}, k$ variable.
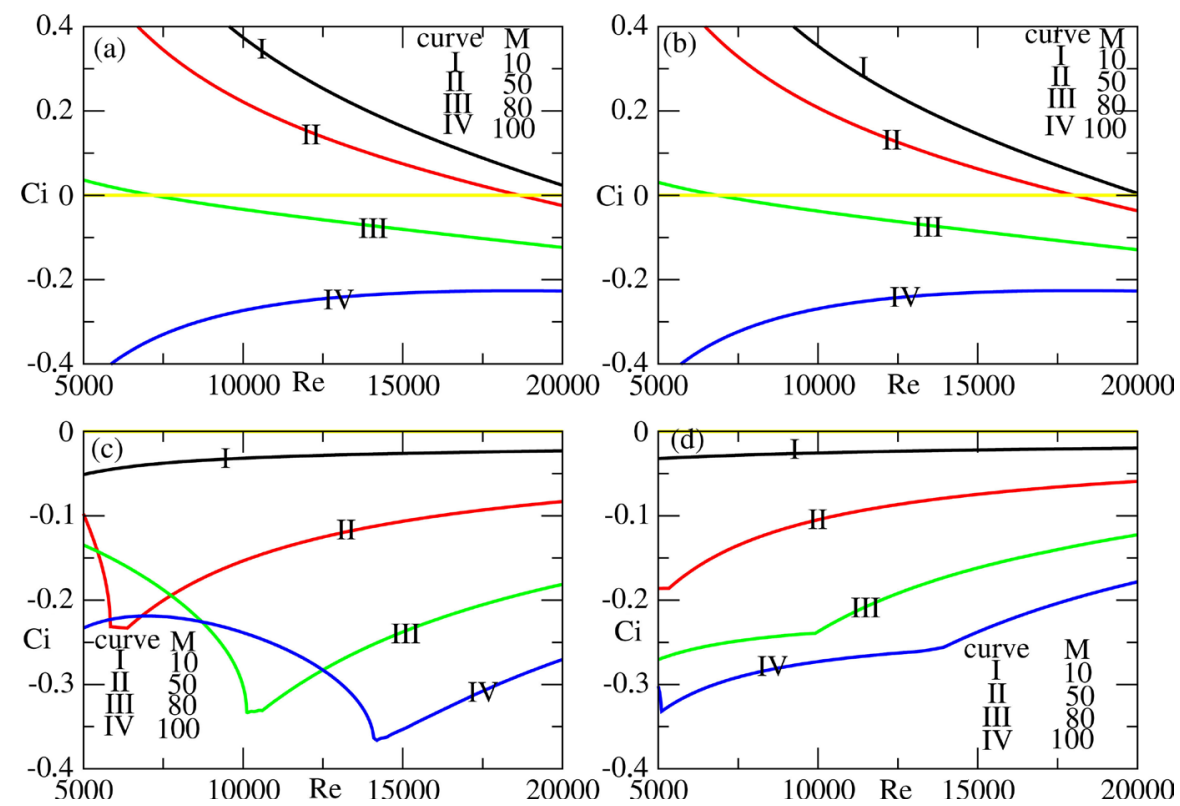

Figure 4. Ci vs. Re for $R_{e \omega}=0, K_{p}=0.045, \theta=0$ and $M, k$ variable.

may be concluded that except the frame $c$ case where, the increasing of $K_{p}$ doesn't contribute to stability for $R_{e}<12500$, the increasing of permeabilty parameter contributes to the flow stability.

Figure 4 shows the effect of Hartmann parameter $M$ on linear temporal stability of viscous incompressible electrically conducting fluid flow for different values of wave number. It is observed that $M$ affects the stability. For $k=1$ and $k=1.02$, frames $a$ and $b$ show that for $M=10$ the flow is unstable (see curves I) and stable for $M=100$ (see curves IV) but for $M=50$ and $M=80$ (curves II, III), we have the transition of the flow (See Table 1 for the critical Reynolds number values). For $k=2$ and $k=3$ (frames $c$ and $d$ ) 
the flow is completely stable. Thus, we may concluded that the Hartmann parameter increasing contributes more to the flow stability.

Figure 5 depicts the effect of phase angle $\theta$ for different values of the wave number on the flow stability. For $k=1$ and $k=1.02$, frames $a$ and $b$ show that the flow is unstable and the instability increases when $\theta$ increases. But, for $k=2$ and $k=3$ the flow is completely stable except the curve IV frame $c$, which presents a transition initialy and stays stable after.

Finally, Figures 6-8 ( $M \neq 0$, electrically conducting fluid) show that for $k=1$ and $k=1.02$, the small injection/suction has no effect on the linear temporal stability of the flow. But for $k=2$ and $k=3$, we remark a little influence of the
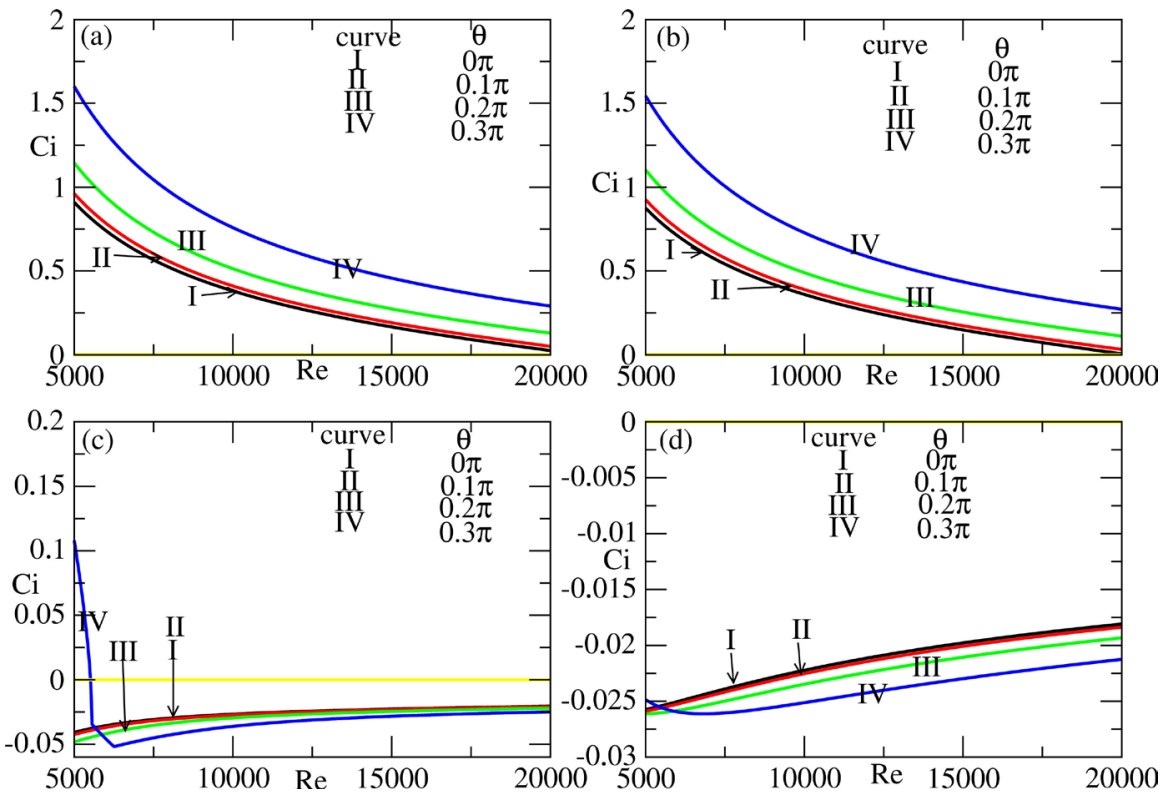

Figure 5. Ci vs. Re for $R_{e \omega}=0, K_{p}=0.045, M=0$ and $\theta, k$ variable.
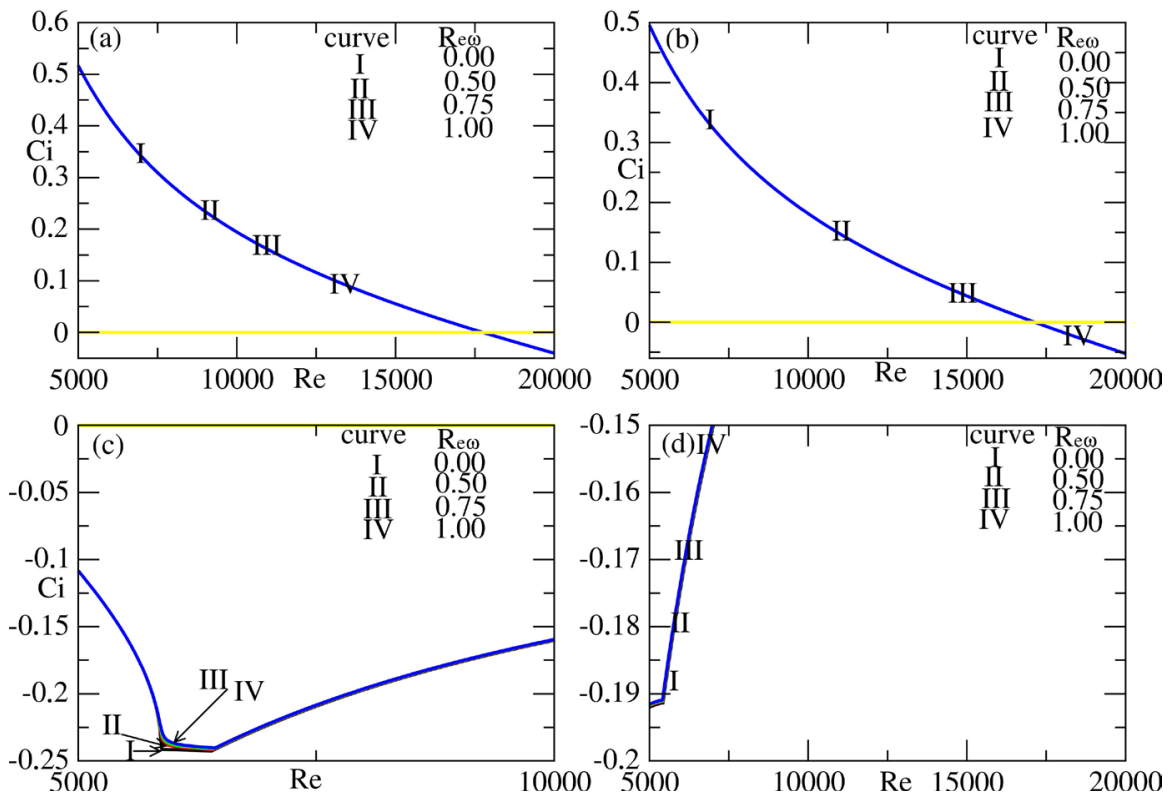

Figure 6. Ci vs. Re for $\theta=0.1 \pi, K_{p}=0.048, M=50$ and $R_{e \omega}, k$ variable. 

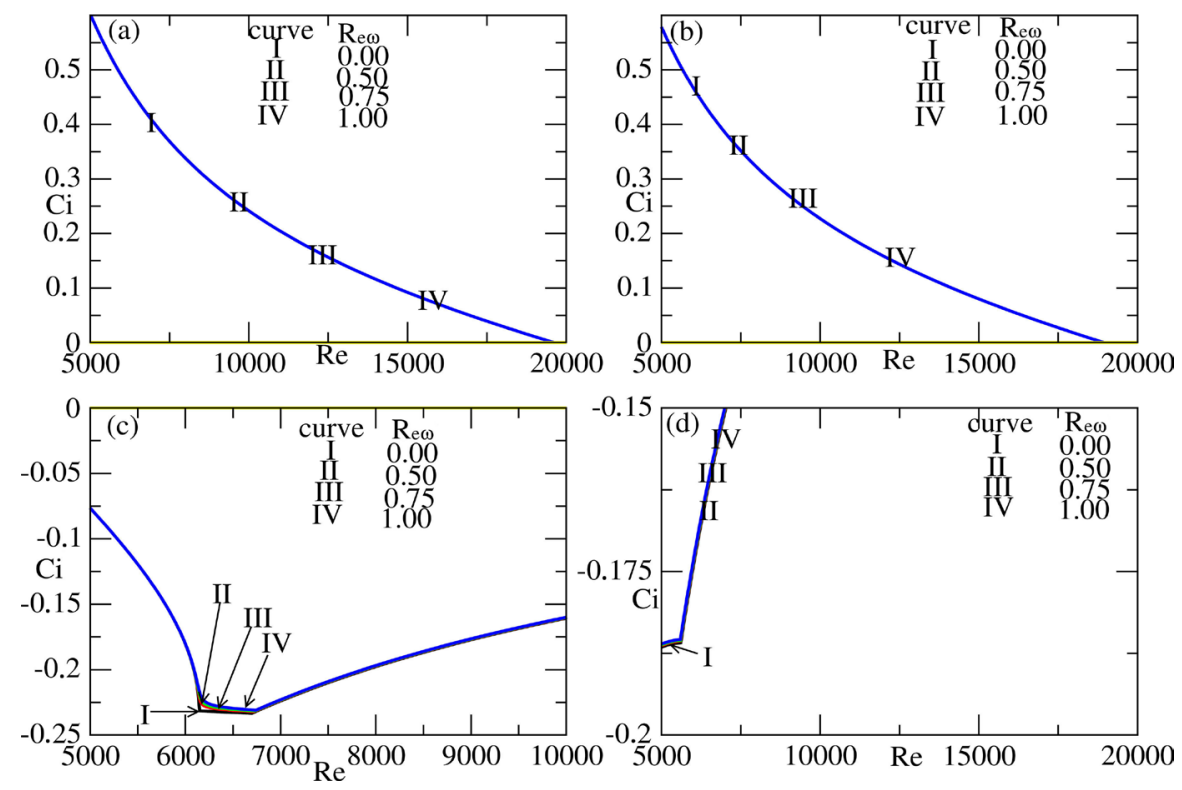

Figure 7. Ci vs. Re for $\theta=0.1 \pi, K_{p}=0.045, M=50$ fixed and $R_{e \omega}, k$ variable.
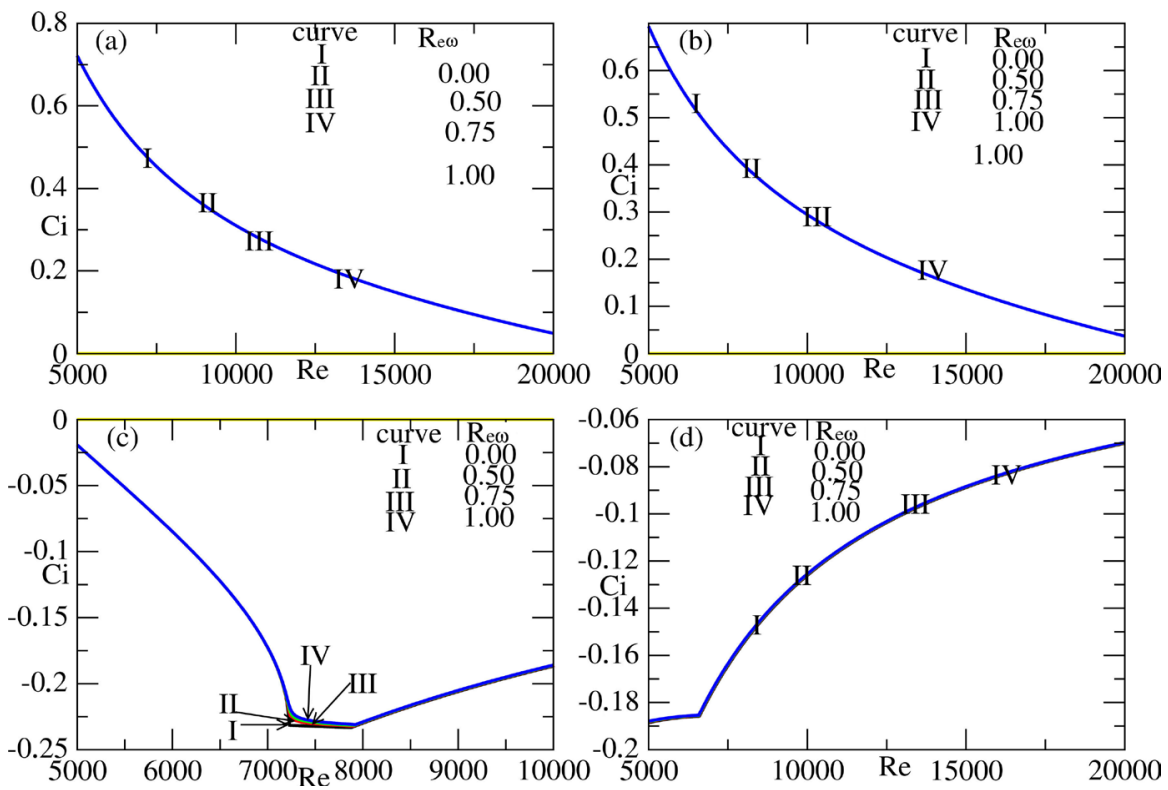

Figure 8. Ci vs. Re for $\theta=0.2 \pi, K_{p}=0.045, M=50$ and $R_{e \omega}, k$ variable.

small injection/suction on the stability only in a small range of $R_{e}$.

The critical Reynolds numbers $R_{e c}$ for which transition occurs are presented in Table 1.

\section{Conclusion}

In this paper, we have investigated the effects of small injection/suction Reynolds number, Hartmann parameter, permeability parameter and wave number on a viscous incompressilbe electrically conducting fluid flow, in a porous parallel plates forming a channel. We have derived the appropriate equation named modified Orr-Sommerfeld equation in order to make the 
Table 1. Table of critical values.

\begin{tabular}{|c|c|c|c|c|c|}
\hline$R_{e \omega}$ & $k$ & $K_{p}$ & $M$ & $\theta(\pi)$ & $R_{e c}$ \\
\hline 0.00 & 1.00 & 0.130 & 00 & 0.00 & 5347 \\
\hline 0.00 & 1.00 & 0.048 & 00 & 0.00 & 19370 \\
\hline 0.00 & 1.02 & 0.130 & 00 & 0.00 & 5139 \\
\hline 0.00 & 1.02 & 0.048 & 00 & 0.00 & 18640 \\
\hline 0.00 & 1.00 & 0.045 & 50 & 0.00 & 18650 \\
\hline 0.00 & 1.00 & 0.045 & 80 & 0.00 & 7178 \\
\hline 0.00 & 1.02 & 0.045 & 50 & 0.00 & 18010 \\
\hline 0.00 & 1.02 & 0.045 & 80 & 0.00 & 6845 \\
\hline 0.00 & 1.00 & 0.045 & 50 & 0.10 & 19610 \\
\hline 0.50 & 1.00 & 0.045 & 50 & 0.10 & 19610 \\
\hline 0.75 & 1.00 & 0.045 & 50 & 0.10 & 19610 \\
\hline 1.00 & 1.00 & 0.045 & 50 & 0.10 & 19610 \\
\hline 0.00 & 1.02 & 0.045 & 50 & 0.10 & 18940 \\
\hline 0.50 & 1.02 & 0.045 & 50 & 0.10 & 18940 \\
\hline 0.75 & 1.02 & 0.045 & 50 & 0.10 & 18940 \\
\hline 1.00 & 1.02 & 0.045 & 50 & 0.10 & 18940 \\
\hline 0.00 & 1.00 & 0.048 & 50 & 0.10 & 17750 \\
\hline 0.50 & 1.00 & 0.048 & 50 & 0.10 & 17750 \\
\hline 0.75 & 1.00 & 0.048 & 50 & 0.10 & 17750 \\
\hline 1.00 & 1.00 & 0.048 & 50 & 0.10 & 17750 \\
\hline 0.00 & 1.02 & 0.048 & 50 & 0.10 & 17140 \\
\hline 0.50 & 1.02 & 0.048 & 50 & 0.10 & 17140 \\
\hline 0.75 & 1.02 & 0.048 & 50 & 0.10 & 17140 \\
\hline 1.00 & 1.02 & 0.048 & 50 & 0.10 & 17140 \\
\hline
\end{tabular}

stability analysis of the flow. Through this approach, we have found that the small injection/suction has a negligible effect on the linear temporal stability of hydromagnetic Couette flow. We noticed that the permeability parameter (Darcy number), the Lorentz force (the Hartmann parameter) and the wave number contribute to the temporal linear stability of hydromagnetic Couette flow. We remarked also that at low wave numbers, the phase angle $\theta$ doesn't contribute to the stability of the fluid flow, but for $k=2$ and $k=3$, the stabilizing effect appears.

\section{Acknowledgements}

The authors would like to thank very much the anonymous referees whose useful criticisms, comments and suggestions have helped strengthen the content and the quality of the paper. 


\section{References}

[1] Ajadi, O.S. (2005) A Note on Unsteady Flow of Dusty Viscous Fluid between Two Parallel Plates. Journal of Applied Mathematics and Computing, 18, 393-403. https://doi.org/10.1007/BF02936582

[2] Das, S.S. (2009) Effect of Suction and Injection on MHD Three Dimensional Couette Flow and Heat Transfer through a Porous Medium. Journal of Naval Architecture and Marine Engineering, 41, 41-51.

[3] Sayed Ahmed, E.M., Hazem, A.A. and Karem, M.E. (2011) Time Dependent Pressure Gradient Effect on Unsteady MHD Couette Flow and Heat Transfer of a Casson Fluid. Journal Engineering, 3, 38-49. https://doi.org/10.4236/eng.2011.31005

[4] Monwanou, A.V. and Chabi Orou, J.B. (2012) The Inviscid Instability in an Electrically Conducting Fluid Affected by a Parallel Magnetic Field. The African Review of Physics, 7, 377-382.

[5] Chand, K., Kumar, R. and Sharma, S. (2012) Hydromagnetic Oscillatory Flow through a Porous Medium Bounded by Two Vertical Porous Plates with Heat Source and Soret Effect. Advances in Applied Science Research, 53, 2169-2178.

[6] Prasad, V.R., Raju, S. and Venkataraman, S. (2012) Unsteaedy Hydromagnetic Flow through a Porous Medium in a Horizontal Channel under Prescribed Discharge with Inclined Magnetic Field. International Journal of Emerging Technology and Advanced Engineering, 2, 277-292.

[7] Shalini, B., Saroa, S.M. and Rajeev, J. (2012) Unsteady Flow of a Dusty Conducting Fluid through Porous Medium between Parallel Porous Plates with Temperature Dependent Viscosity and Heat Source. International Journal of Scientific Research Engineering and Technology, 3, 013-021.

[8] Nayak, A. and Dash, C. (2013) Oscillatory Effect on Magnetohydrodynamic Flow and Heat Transfer in a Rotating Horizontal Porous Channel. Annals of Faculty Engineering Hunedoara-International Journal of Engineering, Tome XI Fascicule 1 ISSN 1584-2665, 199-208.

\section{Scientific Research Publishing}

Submit or recommend next manuscript to SCIRP and we will provide best service for you:

Accepting pre-submission inquiries through Email, Facebook, LinkedIn, Twitter, etc. A wide selection of journals (inclusive of 9 subjects, more than 200 journals)

Providing 24-hour high-quality service

User-friendly online submission system

Fair and swift peer-review system

Efficient typesetting and proofreading procedure

Display of the result of downloads and visits, as well as the number of cited articles

Maximum dissemination of your research work

Submit your manuscript at: http://papersubmission.scirp.org/

Or contact jamp@scirp.org 